\title{
Óxido nítrico inalatório: considerações sobre sua aplicação clínica*
}

\author{
Inhaled nitric oxide: clinical application
}

\author{
Gisele Limongeli Gurgueira ${ }^{1}$, Werther Brunow de CARvalho ${ }^{2}$
}

O objetivo dos autores é relatar alguns aspectos da aplicação clínica e terapêutica do óxido nítrico inalatório em pediatria. As referências foram obtidas através do Medline ${ }^{\circledR}$ utilizando as palavras-chave: óxido nítrico inalatório e pediatria, e cuidados intensivos. O utras bases de pesquisa utilizadas foram obtidas na biblioteca da universidade e em arquivos pessoais. Na última década, os estudos clínicos com o óxido nítrico inalatório comprovaram sua eficácia somente em situações específicas e associação a uma variedade de efeitos adversos. A toxicidade do óxido nítrico inalatório inclui metahemoglobinemia, efeitos citotóxicos pulmonares, produção aumentada de dióxido de nitrogênio e peroxinitrito e alterações no sistema surfactante pulmonar. A administração do óxido nítrico inalatório a pacientes com disfunção ventricular esquerda grave e hipertensão pulmonar deve ser extremamente cautelosa, pois a vasodilatação determina elevação do fluxo sanguíneo pulmonar, podendo aumentar muito a pré-carga. Alguns estudos observaram efeitos clínicos relacionados com a retirada abrupta do óxido nítrico inalatório, como a hipertensão pulmonar rebote. A tualmente, de acordo com a literatura médica, o uso do óxido nítrico inalatório está aprovado na hipertensão pulmonar persistente do neonato (idade gestacional igual a ou acima de 34 semanas) para melhorar a oxigenação e evitar a necessidade de oxigenação por membrana extracorpórea e nas cardiopatias congênitas acompanhadas de hipertensão pulmonar, principalmente durante o pós-operatório imediato. A té hoje algumas pesquisas pediátricas e estudos multicêntricos em adultos não conseguiram demonstrar redução na mortalidade e no tempo de ventilação pulmonar mecânica com o uso do óxido nítrico inalatório na síndrome do desconforto respiratório agudo e na lesão pulmonar aguda. Investigações futuras são necessárias para esse uso. A hipertensão pulmonar persistente é a indicação mais importante para o uso do óxido nítrico inalatório. A Food and Drug Administration não aprova o uso do óxido nítrico inalatório para síndrome do desconforto respiratório agudo em crianças e adultos. (J Pneumol 2003;29(5):325-31)

Descritores - Óxido nítrico/ uso terapêutico. Hipertensão pulmonar. Cuidados críticos. Pediatria.

* Trabalho realizado na U niversidade Federal de São Paulo - Escola Paulista de Medicina.

1. Médica A ssistente da U nidade de Cuidados Intensivos Pediátrica do Hospital São Paulo, do Hospital Santa Catarina e do Hospital do Servidor Público Municipal.

2. Professor Livre-Docente do Departamento de Pediatria da U niversidade Federal de São Paulo/ Escola Paulista de Medicina. Chefe das
The objective of this paper is to report some clinical and therapeutic aspects of inhaled nitric oxide in pediatrics. Some references were obtained from Medline ${ }^{\circledR}$ using the keywords: inhaled nitric oxide and pediatrics, and critical care. Other sources were the University library and personal files. A long the last decade, clinical trials with inhaled nitric oxide demonstrated only a few specific areas of proven efficacy and a variety of possible adverse events. Toxicity related to inhaled nitric oxide included metahemoglobinemia, cytotoxic pulmonary effects, excess production of nitrogen dioxide and peroxynitrite, and injury to the pulmonary surfactant system. The administration of inhaled nitric oxide to patients with severe left ventricular dysfunction and pulmonary hypertension should be cautious, since vasodilatation may increase pulmonary blood flow and lead to excessive preload. Some studies showed the clinical effects related to abrupt nitric oxide withdrawal, including rebound pulmonary hypertension. Current literature supports the therapeutic use of inhaled nitric oxide in persistent pulmonary hypertension of the newborn (gestational age $\geq 34$ weeks) to improve oxygenation and avoid extracorporeal oxygenation; and in congenital cardiopathy accompanied by pulmonary hypertension, especially in the immediate postoperative period. To date, research in pediatrics and multicentre trials in adults with inhaled nitric oxide therapy have failed to show mortality reduction or decrease the amount of time under mechanical ventilation for acute respiratory distress syndrome and acute lung injury. This indication needs further investigations. Persistent pulmonary hypertension is the most important indication for inhaled nitric oxide. The Food and Drug Administration has not approved inhaled nitric oxide in acute respiratory distress syndrome for adults and children.

Key words - Nitric oxide/ therapeutic use. Pediatrics. Pulmonary hypertension. Critical care.

U CIs Pediátricas do H ospital São Paulo, do H ospital Santa Catarina e do Hospital Beneficiência Portuguesa.

Endereço para correspondência - Rua Calógero Calia, 230 - 04250 100 - São Paulo, SP. Tel.: (11) 5058-2753; e-mail: limongeli@uol. com.br; wertherbru.dped@epm.br

Recebido para publicação em 14/1/03. Aprovado, após revisão, em 11/6/03. 


\section{INTRODUÇãO}

Há cerca de 20 anos, o óxido nítrico (NO) era considerado somente um gás nocivo, extremamente tóxico e que existia na natureza por não mais que alguns segundos. Pela sua alta reatividade (trata-se de um radical livre com um elétron extra) é convertido rapidamente, através da reação com oxigênio e água, em nitratos e nitritos.

Entre 1984 e 1987, vários estudos demonstraram que o No é produzido por várias células do organismo, sendo essencial em inúmeras funções orgânicas. ${ }^{(1-5)}$ Nos vasos sanguíneos, sua formação contínua pelas células endoteliais promove o relaxamento da musculatura lisa subjacente, produzindo vasodilatação. ${ }^{(6-8)}$ No sistema imune, macrófagos, quando estimulados, produzem grande quantidade de No, que funciona como uma molécula killer, destruindo células-alvo (cancerosas) e microorganismos. ${ }^{(2,3)}$ O NO atua também em outros sistemas, tais como o sistema nervoso central (SNC), gastrintestinal, respiratório, cardíaco e genitourinário.(2,3) Esses achados levaram a extensa produção científica relacionada ao No.(1)

A utilização do No inalatório em doenças que levavam à hipertensão pulmonar (H P) seria, então, altamente promissora, por seu efeito vasodilatador pulmonar seletivo, melhorando a relação ventilação/ perfusão e aumentando o desempenho cardíaco direito. O s estudos pediátricos desenvolvidos, em sua grande maioria, são com amostras pequenas e avaliaram somente os efeitos hemodinâmicos e na oxigenação com 0 NO inalatório. ${ }^{(1,9-11)}$ A penas dois estudos prospectivos com uma casuística maior, em recém-nascidos, demonstraram de modo claro os efeitos benéficos da inalação do No na síndrome da hipertensão pulmonar persistente do neonato (HPPN), ${ }^{(12,13)}$ resultando na aprovação do uso do No inalatório pela Food and Drug Administration (FDA) para os neonatos de termo e próximos ao termo ( $\geq 34$ semanas). Estudos em outras doenças, como na síndrome do desconforto respiratório agudo (SDRA), não conseguiram demonstrar um efeito benéfico consistente e, portanto, seu uso ainda permanece restrito e com inúmeras questões a serem respondidas. ${ }^{(15-21)}$

\section{TOXICIDADE}

O NO pode produzir diversas substâncias tóxicas. $\mathrm{Na}$ presença de oxigênio $\left(\mathrm{O}_{2}\right)$ é oxidado em dióxido de nitrogênio $\left(\mathrm{NO}_{2}\right)$, um gás altamente citotóxico que em solução aquosa é convertido em ácido nítrico e nitroso. 0 Instituto de Segurança O cupacional e Administração de Saúde dos EUA fixa o limite de 5 ppm de exposição ao $\mathrm{NO}_{2}$ para os trabalhadores. (22) Tem-se observado que grande parte da toxicidade do $\mathrm{NO}$ deve-se à formação do $\mathrm{NO}_{2}$ a partir de sua reação com $0 \mathrm{O}_{2}$.

Durante a utilização do No inalatório para os pacientes com HP, podemos evitar a inalação do $\mathrm{NO}_{2}$ interpondo
Siglas e abreviaturas utilizadas neste trabal ho

CEC - Circulação extracorpórea

CINRGI - Clinical Inhaled Nitric Oxide Research Group Initiative

DNA - Ácido desoxirribonucléico

DPOC - Doença pulmonar obstrutiva crônica

EUA - Estados Unidos da A mérica

FDA - Food and Drug Administration

$\mathrm{FiO}_{2}$ - Fração inspirada de oxigênio

HP - Hipertensão pulmonar

HPP - Hipertensão pulmonar primária

HPPN - Hipertensão pulmonar persistente do neonato

10 - Índice de oxigenação

$\mathrm{N}_{2}$ - Nitrogênio

NADH - Nicotinamida adenina dinucleotídeo

NINOS - Neonatal Inhaled Nitric Oxide Study

NO - Óxido nítrico

$\mathrm{NO}_{2}$ - Dióxido de nitrogênio

NOS - Óxido nítrico sintetase

$\mathrm{O}_{2}$ - Oxigênio

OMEC - Oxigenação por membrana extracorpórea

$\mathrm{PaO}_{2}$ - Pressão arterial de oxigênio

PAP - Pressão arterial pulmonar

PAS - Pressão arterial sistêmica

$P G I_{2}$ - Prostaglandina $I_{2}$

RVP - Resistência vascular pulmonar

SDRA - Síndrome do desconforto respiratório agudo

SNC - Sistema nervoso central

VD - Ventrículo direito

VE - Ventrículo esquerdo

VPM - Ventilação pulmonar mecânica

um sistema com cal sodada no circuito do aparelho de ventilação pulmonar mecânica (VPM), ou utilizando determinadas concentrações de $\mathrm{NO}$ e $\mathrm{O}_{2}$ que levem a uma baixa taxa de conversão para $\mathrm{NO}_{2}$ (a velocidade de oxidação é dependente da concentração de $\mathrm{O}_{2}$ e do quadrado da concentração de NO). Quando o No é armazenado em cilindros com nitrogênio $\left(N_{2}\right)$ puro, não há a formação de $\mathrm{NO}_{2} \cdot{ }^{(1)}$

Em solução aquosa o No reage com radicais superóxidos formando o superoxidonitrito, uma substância citotóxica. Além disso, pode formar complexos com metais, como por exemplo na sua ligação com a hemoglobina formando a nitrosil Fe (II)-hemoglobina e, posteriormente, a metahemoglobina. ${ }^{(1)}$

A metahemoglobinemia ocorre por aumento de produção da metahemoglobina, ou quando a sua redução através da diaforase NADH (metahemoglobina redutase) encontra-se diminuída. De acordo com alguns estudos, os níveis de metahemoglobina não aumentaram significativamente em exposições a baixas concentrações de $\mathrm{NO}_{2} \cdot{ }^{(3,24)}$ Entretanto, indivíduos que apresentam diminuição da atividade da diaforase NADH podem vir a desenvolvê-la. Sabe-se que em recém-nascidos a atividade dessa enzima é normalmente diminuída. ${ }^{(1)}$ 
A lém do seu efeito tóxico quando da reação com outras substâncias, o próprio No pode causar lesão celular direta principalmente através de mutações no ácido desoxirribonucléico (DNA) do núcleo celular. ${ }^{(25)}$

\section{Principais efeitos adversos}

Existem alguns efeitos adversos relacionados à toxicidade do No, como a formação de metemoglobina, ${ }^{(26)}$ efeitos citotóxicos pulmonares devido à formação de radicais livres pelo excesso de dióxido de nitrogênio $\left(\mathrm{NO}_{2}\right)$, produção de peroxinitrito, ou alteração do sistema de surfactante pulmonar. ${ }^{(14)}$ A lém desses, existem outros, como os carcinogênicos, os teratogênicos, os efeitos desconhecidos no pulmão imaturo e os efeitos na hemostasia, como alteração da agregação plaquetária.(27) A pesar de ser prudente considerar o risco de coagulopatia com o uso do No inalatório, o aumento da sua incidência não foi observado por estudos prospectivos randomizados. ${ }^{(28-30)}$

Nos pacientes com disfunção importante de ventrículo esquerdo (VE) e HP, a vasodilatação pulmonar súbita pode aumentar o fluxo sanguíneo, elevando a pré-carga de forma deletéria para um ventrículo previamente comprometido. (26)

Vários estudos demonstraram efeitos indesejáveis com a retirada abrupta do No, tendo sido também observado efeito rebote. ${ }^{(31-36)}$ As razões para que ocorra o efeito rebote ainda não estão bem esclarecidas, mas podem estar relacionadas com a inibição da atividade da óxido nítrico sintetase (NOS). A lguns métodos são utilizados para evitar esse efeito durante a retirada do No inalatório. 0 método mais utilizado é o aumento da fração inspirada de oxigênio $\left(\mathrm{FiO}_{2}\right)$ antes da diminuição do NO. (37) Outros pesquisadores têm utilizado um inibidor da fosfodiesterase, o dipiridamole, para atenuar o efeito rebote durante a retirada do NO inalatório ${ }^{(38,39)}$

\section{NO INALATÓRIO - APLICAÇÕES}

A insuficiência respiratória aguda permanece como um dos principais fatores para a morbidade e a mortalidade em pacientes críticos. A H P contribui para a piora da hipoxemia e da sobrecarga ventricular direita e está associada à taxa de mortalidade elevada em diversas patologias, como a hipertensão pulmonar persistente do neonato (HPPN), a síndrome do desconforto respiratório agudo (SDRA), as cardiopatias congênitas com HP, em pacientes com doença pulmonar obstrutiva crônica (DPOC), hipertensão pulmonar primária (HPP) e outras. Um estudo em pacientes adultos com SDRA demonstrou que o aumento da resistência vascular pulmonar (RVP) correlaciona-se diretamente com a gravidade das alterações nas trocas gasosas. ${ }^{(1)}$ Esses achados levaram a inúmeras pesquisas tentando identificar um vasodilatador pulmonar seletivo. A terapêutica com vasodilatadores intravenosos é limitada, pois não consegue diminuir a RVP sem que cause efeitos colaterais importantes, como a hipotensão sistêmica, que diminui a perfusão coronariana e conseqüentemente piora a função ventricular direita.

0 termo macrosseletividade tem sido utilizado para distinguir os efeitos de uma droga vasodilatadora na vasculatura pulmonar do efeito produzido na circulação sistêmica. A macrosseletividade é determinada pela razão entre a pressão arterial pulmonar (PAP) e a pressão arterial sistêmica (PAS), ou pela razão entre as respectivas resistências. U m vasodilatador pulmonar macrosseletivo deve ser capaz de reduzir a RVP e promover a melhora da função ventricular direita. A tualmente não existe um agente intravenoso que atue somente na vasculatura pulmonar. ${ }^{(1)}$

0 termo microsseletividade tem sido utilizado para distinguir os efeitos de uma droga vasodilatadora na distribuição do fluxo sanguíneo pulmonar (perfusão). Muitos agentes intravenosos utilizados, como o nitroprussiato, pioram as trocas gasosas por levarem à vasodilatação não seletiva das artérias pulmonares, perfundindo áreas não ventiladas, o que resulta em piora da ventilação/ perfusão.(1)

O No inalatório apresenta essas duas propriedades de seletividade. A o contrário dos vasodilatadores sistêmicos, atinge somente os alvéolos que estão sendo ventilados, provocando com isso a vasodilatação pulmonar seletiva. Desviando o fluxo para essas áreas consegue melhorar a oxigenação e também o shunt intrapulmonar. 0 No reage avidamente com a hemoglobina, produzindo compostos inativos (nitrito e nitrato). Desse modo não causa vasodilatação sistêmica e não altera a perfusão coronariana. ${ }^{(1)}$

Apesar do uso do No inalatório parecer benéfico para pacientes que apresentam H P por diversas etiologias, em alguns deles observou-se falha na resposta, qual seja, a ausência de um aumento na pressão arterial de oxigênio $\left(\mathrm{PaO}_{2}\right)$ ou diminuição na RVP. (27)

A tualmente, a FDA aprovou a administração do No inalatório apenas para o tratamento de neonatos a termo e prematuros com idade gestacional igual a ou maior que 34 semanas, com insuficiência respiratória hipoxêmica e evidência de HP clínica ou ecocardiográfica, e para crianças com cardiopatia congênita e H P no manejo pré e pósoperatório. ${ }^{\left({ }^{40)}\right.}$ Os efeitos clínicos benéficos do NO inalatório puderam ser demonstrados apenas em dois estudos clínicos: o grupo Inhaled Neonatal Nitric Oxide Study (NINOS) e o CINRGI, em que houve redução significativa do uso da oxigenação por membrana extracorpórea (OMEC). ${ }^{12,13)}$

\section{Cardiopatias congênitas}

Os pacientes com cardiopatia congênita podem desenvolver H P tanto por doença pulmonar como por hiperresponsividade da vasculatura pulmonar. As crianças com 
obstrução intra-útero ao fluxo sanguíneo pulmonar podem apresentar hipoplasia das unidades alvéolo-capilares. A hipoxemia venosa que acompanha a hipoxemia arterial, nesses pacientes, pode manter a RVP elevada. Por outro lado, um dilema comum na prática clínica dáse na questão da manutenção da RVP elevada para equilibrar as circulações pulmonar e sistêmica, no intuito de manter a oxigenação tecidual adequada nos casos de truncus arteriosus, síndrome do ventrículo esquerdo hipoplásico ou outras variantes de ventrículo único. Nesses pacientes a redução da resistência vascular pulmonar com o uso do No inalatório pode ser fatal. Por isso, o uso do No nas cardiopatias congênitas, principalmente no período neonatal, deve ser considerado somente após um estudo cuidadoso da anatomia e da fisiologia.

As cardiopatias congênitas que cursam com hiperfluxo pulmonar ou obstrução à drenagem das veias pulmonares apresentam hipertrofia e hiperplasia da musculatura vascular e conseqüente vasoconstrição. A H P permanece como importante causa de morbidade e mortalidade no pós-operatório imediato de crianças submetidas a correção cirúrgica da cardiopatia. O No inalatório pode ser utilizado na avaliação da vasorreatividade pulmonar durante o cateterismo, permitindo o planejamento do tratamento cirúrgico mais adequado. Dois grupos de pacientes se beneficiam dessa avaliação: naqueles com ventrículo único em que a anastomose cavopulmonar está indicada, o sucesso da cirurgia depende da manutenção da RVP baixa; o segundo grupo de pacientes é aquele em que há hiperfluxo pulmonar devido ao shunt esquerdo-direito, em que, com o passar do tempo, há a hiperplasia da camada muscular dos capilares pulmonares, o que a torna menos responsiva aos agentes farmacológicos.

Em crianças com cardiopatias diversas, respirando espontaneamente, com o uso do No inalatório (até $80 \mathrm{ppm}$ ) observou-se redução significativa da RVP durante o cateterismo, sem causar hipotensão sistêmica ou elevação significativa nos níveis de metahemoglobina. ${ }^{(41)}$ Resultado semelhante foi observado em pacientes pediátricos com cardiopatia congênita e HP, na avaliação pré-operatória da HP com o uso de No inalatório na dose de 10 e $20 \mathrm{ppm} .{ }^{(42)}$

$O$ aumento da RVP é freqüente nos pacientes cardiopatas e esta pode ser exacerbada com o emprego da circulação extracorpórea (CEC). Concomitante ao estado patológico preexistente, ocorre uma disfunção do ventrículo direito (VD) após a CEC, devida à preservação inadequada do VD durante a cirurgia, à produção de vasoconstritores pulmonares como a tromboxana $\mathrm{A}_{2}$, resultante da agregação plaquetária e leucocitária, ou pela diminuição de vasodilatadores endógenos como o óxido nítrico (NO). A H P contribui para a piora da hipoxemia e da sobrecarga do VD. $O$ tratamento convencional após a cirurgia consis- te em hiperventilação, alcalinização, aumento da $\mathrm{FiO}_{2}$, uso de inotrópicos e vasodilatadores. $O$ uso do No inalatório mostrou ser benéfico e eficaz nesses pacientes. ${ }^{(43-45)}$

Miller et al., utilizando baixas concentrações de No $(2$, 10 e 20 ppm), obtiveram redução significante da pressão arterial pulmonar em 10 lactentes no pós-operatório de cirurgia cardíaca com alto risco para HP. ${ }^{(46)}$

U m estudo comparando os efeitos da hiperventilação e do uso do No inalatório, em pacientes pediátricos no pósoperatório de correção de cardiopatia com HP, demonstrou que as duas técnicas são efetivas em diminuir a RVP e a PAP. O uso do No inalatório apresentou vantagem em relação à hiperventilação, pois não houve diminuição do débito cardíaco e aumento da resistência vascular sistêmica (RVS). ${ }^{(47)}$

A tualmente a FDA aprova a utilização do No inalatório para os pacientes pediátricos submetidos a cirurgia cardíaca e para o manejo pré-operatório de cardiopatias com HP.

\section{Lesão pulmonar aguda/síndrome do desconfor- to respiratório agudo}

A hipoxemia e a HP estão presentes na SDRA e a gravidade de cada uma está intimamente relacionada com a mortalidade. (16-19) A HP na SDRA é causada por vasoconstrição ativa (devida a hipóxia alveolar, mediadores circulantes, aumento da liberação de mediadores de vasoconstrição como a tromboxana, ou diminuição de mediadores de vasodilatação como o No endógeno), ou por fatores mecânicos (tromboembolismo, compressão vascular por edema ou pressão alveolar elevada). A hipoxemia é causada por alteração na ventilação/ perfusão, shunt intrapulmonar, ou shunt anatômico (forame oval patente). 0 uso de vasodilatadores intravenosos como a nitroglicerina ou prostaglandinas $\left(\mathrm{PGI}_{2}\right)$ resulta em diminuição leve na PAP, mas com grande diminuição na PAS e na oxigenação arterial.(48)

Estudos em adultos e pediátricos demonstraram que 0 No inalatório ocasiona melhora da oxigenação nos pacientes com lesão pulmonar aguda. ${ }^{(31-33)}$ Os efeitos benéficos potenciais do NO na SDRA incluem diminuição na RVP, redução do shunt intrapulmonar, melhora das funções ventriculares direita e esquerda, redução de barotrauma e toxicidade pelo oxigênio por permitir a diminuição dos parâmetros da VPM. A pesar disso, estudos multicêntricos em adultos não conseguiram demonstrar melhora na duração da VPM ou na sobrevida. ${ }^{15,17)}$ Os pacientes apresentaram aumento da oxigenação após o início da inalação do No, mas a resposta foi transitória, não permitindo a redução da VPM ou da $\mathrm{FiO}_{2}$. A chados similares foram demonstrados em outros estudos, incluindo pediátricos, como pode ser observado na Tabela 1.(49) 
O uso do No inalatório nos pacientes com SDRA não deve ser indiscriminado, mas pode ser justificado em pacientes nos quais a melhora da oxigenação pode manter a estabilidade clínica durante a fase aguda da disfunção respiratória hipoxêmica (terapêutica de resgate). Também pode ser utilizado como medida coadjuvante para diminuição da RVP quando é aplicada a estratégia ventilatória com a hipercapnia permissiva. (50)

A té o presente momento, o uso do No inalatório não está aprovado pelo FDA para a SDRA. Em relação a sua eficácia, podemos considerá-lo como possivelmente efetivo, mas seu uso deverá ser extremamente criterioso.

\section{O utras aplicações}

O uso do no inalatório foi testado em outras doenças, mas os resultados não parecem promissores. Em pacientes com DPOC não foi observada melhora significativa na oxigenação que justifique o emprego do No inalatório. ${ }^{(49)}$ Em pacientes pediátricos com hérnia diafragmática a aplicação de No não diminuiu a necessidade de OMEC ou a mortalidade. ${ }^{(49)}$

Pacientes com doenças cardíacas que ocasionam hipertensão pulmonar também podem ser beneficiados com 0 uso do No inalatório. Um estudo abordando os efeitos hemodinâmicos provocados pelo no inalatório em pacientes com HP após valvuloplastia mitral mostrou que houve diminuição na PAP e RVP, e melhora na saturação venosa mista de oxigênio, sem efeitos hemodinâmicos indesejáveis. (52) Pacientes com insuficiência cardíaca direita podem beneficiar-se do uso do No inalatório, pois a diminuição da RVP melhora o desempenho cardíaco. Pode ser utilizado também na avaliação diagnóstica dos pacien- tes candidatos a transplante cardíaco, observando-se a resposta da vasculatura pulmonar com o uso do No inalatório.

Outras possíveis indicações, porém muito pouco estudadas, são: edema pulmonar das grandes altitudes, hemorragia pulmonar, embolia pulmonar e outras.

\section{Contra-indicações}

O uso do No está contra-indicado para recém-nascidos com cardiopatias dependentes de shunt direito-esquerdo, e em presença de deficiência de metahemoglobina redutase congênita ou adquirida.

\section{Precauções}

Deve haver precaução com o uso de No: em presença de anemia, trombocitopenia, leucopenia ou distúrbios de coagulação; em presença de edema pulmonar ou infecção pulmonar aguda; e nos pacientes com disfunção ventricular esquerda grave, os quais somente poderão receber o No inalatório em combinação com outros agentes que melhorem o desempenho ventricular esquerdo.

\section{CONCLUSÃo}

Com base nas evidências clínicas atuais, a indicação e a utilização do No inalatório ficam restritas as duas entidades: hipertensão pulmonar persistente do recém-nascido e hipertensão pulmonar acompanhada de hipoxemia e disfunção de ventrículo direito nas cardiopatias congênitas, principalmente no pós-operatório imediato. No Brasil não existe autorização governamental para a utilização do No inalatório, sendo seu emprego limitado a protocolos de estudos clínicos.

TABELA 1

Resumo de alguns estudos realizados em pacientes pediátricos com SD RA

\begin{tabular}{|c|c|c|c|c|c|}
\hline Autor & Data & População & Dose NO & Mortalidade geral & Achados \\
\hline Abman et al. & 1994 & $\begin{array}{c}10 \text { SDRA } \\
7 \text { pneumonite viral }\end{array}$ & 10ppm & $50 \%$ & $\begin{array}{l}\text { Melhora aguda nas trocas } \\
\text { gasosas e diminuição da VPM }\end{array}$ \\
\hline Day et al. & 1996 & 19 SDRA & 11 ou $60 p p m$ & $45 \%$ & $\begin{array}{l}\text { Melhora na } \mathrm{PaO}_{2} / \mathrm{FiO}_{2} \\
\text { Diminuição no IRVP }\end{array}$ \\
\hline Nakagawa et al. & 1997 & 14 SDRA & 10-80ppm & $38 \%$ & $\begin{array}{l}\text { Diminuição significativa no IO } \\
\text { Dose máxima efetiva = } 20 \mathrm{ppm} \\
\text { Diminuição no IRVP }\end{array}$ \\
\hline Goldman et al. & 1997 & 30 SDRA & 20ppm & $60 \%$ & $\begin{array}{l}\text { Diminuição significativa no } 10 \\
\text { Pacientes com }<15 \% \text { de } \\
\text { resposta faleceram } \\
\text { Mortalidade mínima com } \\
\text { resposta }>30 \%\end{array}$ \\
\hline Day et al. & 1997 & 24 SDRA & 10ppm & $46 \%$ & $\begin{array}{l}\text { Aumento agudo no } 10 \text {, não persistiu } \\
\text { após } 24 \text { horas do tratamento }\end{array}$ \\
\hline
\end{tabular}




\section{REFERÊNCIAS}

1. Nelin LD, Hoffman GM. The use of inhaled nitric oxide in a wide variety of clinical problems. Pediatr Clin North Am 1998;45:531-48.

2. Granger $D L$, Lehninger $A L$. Sites of inhibition of mitochondrial electron transport in macrophage injured neoplastic cells. J Cell Biol 1982; 95:527-35.

3. Mulsch A, Hauschildt S, Bessler WG. Synthesis of nitric oxide in the cytosol of porcine aortic endothelial cells and murine bone marrow macrophages: detection by activation of purified soluble guanylate cyclase. In: Moncada S, Higgs SA, editors. Nitric oxide from L-arginine: a bioregulatory system. Amsterdan: Elsevier, 1990;235-42.

4. McCall T, Vallance P. Nitric oxide takes centre-stage with newly defined roles. Trends Pharmacol Sci 1992;13:1-6.

5. Moncada S, Palmer RMJ, Higgs EA. Nitric oxide: physiology, pathophysiology and pharmacology. Pharmacol Rev 1991;43:109-42.

6. Ignarro LJ . Endothelium-derived nitric oxide: actions and properties. FASEB J 1989;3:31-6.

7. Änggärd E. Nitric oxide: mediator, murderer, and medicine. Lancet 1994;343:1199-206.

8. Forstermann U. Properties and mechanisms of production and action of endothelium-derived relaxing factor. J Cardiovasc Pharmacol 1986; 8 (Suppl 10):S45-51.

9. Roberts J D, Poloner DM, Lang P, Zapol WM. Inhaled nitric oxide in persistent pulmonary hypertension of the newborn. Lancet 1992;340: 818-9.

10. Kinsella J P, Neish SR, Shaffer E, Abman SH. Low-dose inhalational nitric oxide in persistent hypertension of the newborn. Lancet 1992; 340:819-20.

11. A bman SH, Griebel J L, Parker DK, Schmidt J M, Swanton D, Kinsella $J$ P. A cute effects of inhaled nitric oxide in children with severe hypoxemic respiratory failure. J Pediatr 1994;124:881-8.

12. Roberts J D J F, Fineman J R, M orin FC $3^{\text {rd }}$, Shaul PW, Rimar S, Scheiber $M$, et al. Inhaled nitric oxide and persistent pulmonary hypertension of the newborn. N Engl J Med 1997;336:605-10.

13. Group NINOS. Inhaled nitric oxide in full-term and nearly full term infants with hypoxic respiratory failure. N Engl J Med 1997;336:597604.

14. Schechter NA, Gladwin MT, Cannon RO 3rd. NO solutions? J Clin Invest 2002;109:1149-51.

15. Michael J R, Barton RG, Saffle J R, Mone M, Markewitz BA, Hillier K, et al. Inhaled nitric oxide versus conventional therapy: effect on oxygenation in ARDS. Am J Respir Crit Care Med 1998;157:1372-80.

16. Barnes SJ . Nitric oxide in acute respiratory distress syndrome. Enhancing our knowledge at the bench. Crit Care Med 1998;26:115758.

17. Troncy E, Collet JP, Shapiro S, Guimond J G, B lair L, Ducruet T, et al. Inhaled nitric oxide in acute respiratory distress syndrome: a pilot randomized controlled study. Am J Respir C rit Care Med 1998;157:14838.

18. Mattay MA, Pittet J F, J ayr C. J ust say NO to inhaled nitric oxide for acute respiratory distress syndrome. Crit Care Med 1998;26:1-2.

19. Tibby S, Shemie SD. Low-dose inhaled nitric oxide and oxygenation in pediatric hypoxic respiratory failure. Wrong bullet, wrong target. Crit Care Med 1999;27:871-2.

20. Cheifetz I. Inhaled nitric oxide: plenty of data, no consensus. C rit Care Med 2000;28:902-4.

21. Robin $E, H$ addad $E$, Vallet $B$. Le monoxyde d'azote inhalé en période périopératoire et en réanimation. Ann Fr A nesth Reanim 2002;21: 581-90.

22. NIOSH Recommendations for occupational safety and health standards. MMWR Morb Mortal Wkly Rep 1988;37(Suppl 7):1-29.
23. Von Nieding $G$, Wagner $H$, Kockler $H$. Investigation of the acute effects of nitrogen monoxide on lung function in man. Staub-Reinhalt Luft 1975;35:175-8.

24. Beutler E. Methemoglobinemia and sulfhemoglobinemia. In: Williams WJ, Beutler E III, Erslev AJ, et al. Hematology. $2^{\text {nd }}$ ed. New York: McGraw-Hill, 1977;491-4.

25. N guyen $T$, Brunson $D, C$ respi $C L$, et al. DNA damage and mutation in humans cells exposed to nitric oxide in vitro. Proc Natl A cad Sci USA 1992;89:3030-4.

26. Rimar $\mathrm{S}$, Gillis $\mathrm{CN}$. Selective pulmonary vasodilation by inhaled nitric oxide is due to hemoglobin inactivation. Circulation 1993;88:2884-7.

27. Hess DR. Adverse effects and toxicity of inhaled nitric oxide. Respir Care 1999;44:315-30.

28. Kinsella J P, Walsh WF, Bose CL, Gerstmann DR. Inhaled nitric oxide in premature neonates with severe hypoxaemic respiratory failure: a randomized controlled trial. Lancet 1999;354:1061-5.

29. Davidson D, Barefield ES, Kattwinkel J, Dudell G, Damask M, Straube $R$, et al. Inhaled nitric oxide for the early treatment of persistent pulmonary hypertension of the term newborn: a randomized, doublemasked, placebo-controlled, dose-response, multicenter study. Pediatrics 1998;101(3 Pt 1):325-34.

30. Rossaint R, Falke KJ , Lopez F, Slama K, Pison U, Zapol WM. Inhaled nitric oxide for the adult respiratory distress syndrome. N Engl J Med 1993;328:399-405.

31. Gerlach H, Pappert D, Lewandowisk K, Rossaint R, Falke KJ . Long term inhalation with evaluated low doses of nitric oxide for selective improvement of oxygenation in patients with adult respiratory distress syndrome. Intensive Care Med 1993;19:443-9.

32. Bigatello LM, Huford WE, Kacmarec RM, Roberts JD, Zapol WM. Prolonged inhalation of low concentrations of nitric oxide in patients with severe adult respiratory distress syndrome. Effects on pulmonary hemodynamics and oxygenation. A nesthesiology 1994;80:761-70.

33. Miller OI, Tang SF, Keech A, Celermajer DS. Rebound pulmonary hypertension on withdrawal from inhaled nitric oxide (letter). Lancet 1995;346:51-2.

34. Lavoie A, Hall J B, OIson DM, Wylam ME. Life-threatening effects of discontinuing inhaled nitric oxide in severe respiratory failure. Am J Respir Crit Care Med 1996;153(6 Pt 1):1985-7.

35. Atz AM, A datia I, Wessel DL. Rebound pulmonary hypertension after inhalation of nitric oxide. Ann Thorac Surg 1996;62:1759-64.

36. Aly H, Sahni R, Wung J T. Weaning strategy with inhaled nitric oxide treatment in persistent pulmonary hypertension of the newborn. Arch Dis Child Fetal Neonatal Ed 1997;76:F118-F122.

37. Al-Alaiyanm S, Al-O mran A, Dyer D. The use of phosphodiesterase inhibitor (dipyridamole) to wean from inhaled nitric oxide. Intensive Care Med 1996;22:1093-5.

38. Ivy DD, Kinsella J P, Ziegler J W, A bman SH. Dipyridamole attenuates rebound pulmonary hypertension after inhaled nitric oxide withdrawal in postoperative congenital heart disease. J Thorac Cardiovasc Surg 1998;115:875-82.

39. Hurford WE, Bigatello LM. NO-body's perfect. Anesthesiology 2002; 96:1285-7.

40. Roberts J D J r, Lang P, Bigatello ML, Vlahakes GJ , Zapol MW. Inhaled nitric oxide in congenital heart disease. Circulation 1993;87:447-53.

41. Carvalho WB, Carvalho ACC, Gurgueira GL, Ikeda AM, Lee JH, Almeida DR. Inhaled nitric oxide and high concentrations of oxygen in pediatrics patients with congenital cardiopathy and pulmonary hypertension: report of five cases. São Paulo Med J 1998;116:1602-5.

42. Murthy KS, Rao SG, Prakash KS, Robert C, Dhinakar S, Cherian KM. Role of inhaled nitric oxide as a selective pulmonary vasodilator in pediatric cardiac surgical practice. Indian J Pediatr 1999;66:357-61. 
43. J ournois $D$, Poulard $P$, Mauriat $P$, Mallhère $T$, Vouhè $P$, Safran $D$. Inhaled nitric oxide as a therapy for pulmonary hypertension after operations for congenital heart defects. J Thorac Cardiovasc Surg 1994; 107:1129-35.

44. Schranz D, Huth R, Wippermann F, Ritzerfeld S, Schmitt FX, O elert $\mathrm{H}$. Nitric oxide and prostacyclin lower suprasystemic pulmonary hypertension after cardiopulmonary bypass. Eur J Pediatr 1993;152: 793-6.

45. Miller OJ, Celemajer DS, Deanfield JE, Macrae DJ. Very low dose inhaled nitric oxide: a selective pulmonary vasodilator after operations for congenital heart disease. J Thorac Cardiovasc Surg 1994;108: 487-94.

46. Morris K, Beghetti M, Petros A, Adatia I, Bohn D. Comparison of hyperventilation and inhaled nitric oxide for pulmonary hypertension after repair of congenital heart disease. Crit C are Med 2000;28:29748.
47. Sitbon $O$, Brenot $F$, Denjean A, Bergeron A, Parent $F$, Azarion $R$, et al. Inhaled nitric oxide as a screening vasodilator agent in primary pulmonary hypertension. A dose-response study and comparison with prostacyclin. Am J Respir Crit Care Med 1995;151:384-9.

48. Thompson J, Bateman ST, Betit P. Pediatric applications of inhaled nitric oxide. Respir Care 1999;44:177-83.

49. Hammer J. Acute lung injury: pathophysiology, assessment and current therapy. Pediatr Resp Rev 2001;2:1-18.

50. Adnot S, Kouyoumdjian C, Defouilloy C, Andrivet P, Sediame S, Herigault $R$, et al. $H$ emodynamic and gas exchange responses to infusion of acetylcholine and inhalation of nitric oxide in patients with chronic obstructive lung disease and pulmonary hypertension. Am J Respir Dis 1993;148:310-6.

51. Girard C, Lehot J, Pannetier J, Filley S, French P, Estanove S. Inhaled nitric oxide after mitral valve replacement in patients with chronic pulmonary artery hypertension. Anesthesiology 1992;77:880-3. 